\title{
Aprendizaje colaboratívo y Competencias Investigatívas de los estudiantes del décimo ciclo, Universidad Nacional "Federico Villarreal” - Lima, 2018
}

\section{Collaborative Learning and Investigative Competences of the students of the tenth cycle, National University "Federico Villarreal" - Lima, 2018}

Efraín Valverde Arévalo, Abelardo Rodolfo Campana Concha

\begin{abstract}
RESUMEN
Objetivo: Establecer la relación de las variables Aprendizaje Colaborativo y Competencias Investigativas en el contexto en el cual se desarrollan los estudiantes del décimo ciclo, Universidad Nacional “Federico Villarreal”, 2018. Materiales y Métodos: El proceso de investigación tiene un enfoque cuantitativo de diseño correlacional. La muestra fue de 128 participantes, a quienes se les aplicó un cuestionario y un test de conocimientos. Se utilizó el estadístico SPSS, con un margen de error al 5\%. Resultados: La hipótesis general estableció una correlación de Rho = 0,804 que señala al aprendizaje colaborativo como indiferente sobre las competencias investigativas como regular. Las hipótesis específicas hallaron correlaciones de $\mathrm{Rho}=0,401, \mathrm{Rho}=0,613$ y $\mathrm{Rho}=0,559$ con ello se pone en manifiesto que la interdependencia positiva, la responsabilidad individual y de equipo, y la interacción estimuladora inciden de forma poca eficiente en las competencias investigativas, cuyas calificaciones son regulares. Conclusión: Se determinó una correlación muy buena, que hace referencia a las repercusiones del aprendizaje colaborativo señalado mayoritariamente como indiferente, sobre las competencias investigativas cuyas calificaciones destacan por su nivel regular. Concluyendo que las actividades académicas en grupo no son aplicadas en base a las necesidades investigativas durante la formación de los estudiantes.
\end{abstract}

Palabras clave: Aprendizaje colaborativo, competencias investigativas, responsabilidad individual, responsabilidad individual y de equipo, interacción estimuladora

\section{ABSTRACT}

Objective: To establish the relationship of the variables Collaborative Learning and Investigative Competences in the context in which the students of the tenth cycle are developed, National University "Federico Villarreal", 2018. Materials and Methods: The research process has a quantitative design approach correlational The sample consisted of 128 participants, who were given a questionnaire and a knowledge test. The SPSS statistic was used, with a margin of error of $5 \%$. Results: The general hypothesis established a correlation of Rho $=0,804$ that points to collaborative learning as indifferent about research competences as regular. The specific hypotheses found correlations of Rho $=0,401, \mathrm{Rho}=0,613$ and $\mathrm{Rho}=0,559$. This shows that positive interdependence, individual and team responsibility, and stimulating interaction have an inefficient impact on competencies. investigative, whose grades are regular. Conclusion: A very good correlation was determined, which refers to the repercussions of collaborative learning, mostly indicated as indifferent, on research competencies whose grades stand out for their regular level. Concluding that the group academic activities are not applied based on the research needs during the training of the students.

Keywords: Collaborative learning, investigative competencies, individual responsibility, individual and team responsibility, stimulating interaction

\section{INTRODUCCIÓN}

Los institutos de educación superior se han convertido en una alternativa atractiva por los jóvenes que terminar sus estudios básicos, puesto que en un promedio de tres años pueden insertarse en el mercado laboral, sin embargo, un informe realizado por el portal web de la Agencia Andina (2018) indica que de los casi dos millones de jóvenes que cursan estudios superiores, solo uno de cada cuatro elige una carrera técnica, pese a que el Perú es un país con mayor requerimiento de profesionales en ese campo, alertando incluso la necesidad de los empresarios de recurrir a la empleabilidad de profesionales técnicos en el exterior en un futuro próximo.

Sumado a lo anterior señalado, una entrevista publicado en el diario Correo (2019) indica que el mercado laboral en el país requiere incluir 300 mil profesionales técnicos para poder sostener el crecimiento de la economía, pero solo se cubren 100 mil en promedio, en otras palabras se necesita más profesionales técnicos, pero no solo que egrese sino la institución a cargo le brinde los conocimientos y las herramientas académicas de necesarias para cumplir con su labor profesional, esta necesidad de mejorar la educación de educación técnica se refleja la Ley de Institutos y Escuelas de Educación Superior y de la Carrera Pública de sus Docentes $N^{\circ}$ 30512 , en la que se señala que la finalidad de estas instituciones son:

a) Formar a personas en los campos de la ciencia, la tecnología y la docencia, para contribuir con su desarrollo individual, social inclusivo y su adecuado desenvolvimiento en el entorno laboral regional, nacional y global. b) Contribuir al desarrollo del país y a la sostenibilidad de su crecimiento a través del incremento del nivel educativo, la productividad y la competitividad. c) Brindar una oferta formativa de calidad que cuente con las condiciones necesarias para responder a los requerimientos de los sectores productivos y educativos. d) Promover el emprendimiento, la innovación, la investigación aplicada, la educación permanente y el equilibrio entre la oferta formativa y la demanda laboral".

En otras palabras, el Estado está exigiendo mayor compromiso formativo de los institutos, que se ajuste a las necesidades laborales de los estudiantes. Desde la insistencia del Ministerio de Educación para el licenciamiento, estas instituciones están tratando de solventar dichos inconvenientes, sin embargo, aún no experimentamos grandes cambios. Un punto importante que notamos en dicha ley, es que se busca direccionar a los futuros técnicos a una visión científica y tecnología, lo que nos alerta la necesidad de mejorar los aspectos en el área investigativa.

Al presentarse inconvenientes en la formación profesional técnico, también incide en la decisión de los jóvenes para decidir por dichas entidades, puesto que direccionan una atención a una carrera universitaria como una opción más ventajosa y completa para su futuro laboral, según Andina (2018) el 60\% de los egresados de secundaria, entre los 17 y 20 años, es decir, seis de cada 10 alumnos, no sigue estudios superiores y el resto recibe este tipo de enseñanza años después u opta por incorporarse a la vida laboral sin estudiar alguna carrera profesional.

Otro aspecto que hace referencia el SINEACE (2015) es que, ante la necesidad de un profesional técnico, suele brindarle la oportunidad a personal con estudios básicos que con la práctica se va acostumbrando a dicha labor, detallando que de cada cuatro personas de la PEA que trabajan como técnicos en el Perú sólo dos han estudiado una carrera en Institutos de educación superior tecnológica. Según la Encuesta Nacional de Hogares sobre Condiciones de Vida y Pobreza realizado por el INEI (2011) existían un promedio de 2, 343, 451 
profesionales de educación superior tecnológica. Según Is encuesta nacional de hogares sobre condiciones de vida y pobreza realizado por el INEI (2011) existían un promedio de 2,343, 451 profesionales de educación superior técnica, mientras que 688928 trabajan como técnicos, con un cruce de información entre ambas cantidades solo el 348092 estudiaron una carrera técnica y trabajan como técnicos al mismo tiempo, puesto que también existen profesionales técnicos que egresaron, pero no ejercen su profesión representados en el $16.7 \%$ del total inicialmente mencionado.

Al ver dichos cambios en las necesidades técnicas, las necesidades laborales, vemos con mayor interés en implementar metodologías académicas, en este caso estamos optando por las actividades colaborativas para fortalecer los conocimientos de los egresados en base a un desarrollo científico que tengan como resultado profesionales totalmente calificado con un nivel de análisis que no lo aminore frente a profesionales técnicos, aunque dichos objetivos pueden resultar a largo plazo, es necesario identificar los aspectos que dificultad este avance del profesional técnico.

Por ello, la investigación se encuentra justificada mediante la justificación práctica, ya que permitirá fomentar trabajos grupales en más asignaturas de la institución educativa para reforzar los conocimientos con respecto al proceso de investigación, monitoreando el nivel de participación de los integrantes del grupo. En lo social, conseguirá que los docentes y directivos tengan mayor comunicación con respecto a las estrategias y metodologías de trabajo en clases, asimismo de la posibilidad de incluir actividades adicionales que refuercen las competencias investigativas. En lo pedagógico, incentivará a los docentes a motivar a los estudiantes a participar en clases y aportar con sus puntos de vista ya sea en las actividades grupales o individuales, asimismo apoyarlos a la lectura de bibliografía necesaria para mejorar sus conocimientos con respecto al proceso investigativo.

Es importante mencionar algunos antecedentes que fortalecen esta investigación, como la de Álvarez (2015) quien en su investigación titulada "Aprendizaje colaborativo mediado por TIC en la enseñanza universitaria: un acercamiento a las percepciones y experiencias de profesores y alumnos de la Universidad Autónoma de Chihuahua" se concluyó que el 91\% del profesorado, las estrategias colaborativas sí promueven una mayor interacción con sus alumnos, en donde los estudiantes comunican que, si bien se requiere de guía y seguimiento docentes, la interacción depende de la confianza hacia el profesor, y que lamentablemente, es común que el docente intervenga poco durante el desarrollo del trabajo. En este punto, distingue nuevamente una diferencia entre los alumnos de licenciatura y los de posgrado. Los estudiantes de licenciatura consideran que el trabajo colaborativo no incrementa la interacción estudiante-docente, ya que el involucramiento del profesor en la realización del trabajo es poco o nulo en la mayoría de los casos. Por último, detalla que las dificultades de los estudiantes en el aprendizaje colaborativo se reportan son: diferencias en la participación y contribución entre los miembros del equipo, problemas de tiempo y distancia para reunirse, desacuerdos con los compañeros, así como falta de guía y seguimiento docentes.

Asimismo, identificamos que las investigaciones de Páez (2016) titulada "Desarrollo de competencias investigativas basadas en la concepción sistémica de ambiente, en estudiantes de la escuela Normal Superior de Montería" del cual se planteó como objetivo diseñar una estrategia didáctica basada en la concepción sistémica de ambiente que contribuya al desarrollo de competencias investigativas ambientales en los estudiantes del programa de formación complementaria, a partir del tratamiento de los residuos sólidos generados en la Institución. En las conclusiones se destaca que las distintas actitudes frente al manejo y uso inadecuado de residuos son consecuencia de un consumismo indiscriminado de productos por parte de los diversos actores de la institución, un uso inadecuado de las sillas y pupitres y a los restos de especies vegetales que se dispersan en los patios escolares, algunos por cumplir su ciclo de vida y otros por la poda asistida que se les práctica generando abundancia de residuos orgánicos.

En cuanto al objetivo central de la investigación se centra en analizar la relación entre el Aprendizaje Colaborativo con las Competencias Investigativas. Con respecto a los objetivos específicos se plantea: Identificar la relación entre la Interdependencia Positiva, Responsabilidad Individual y de Equipo, e Interacción Estimuladora con las Competencias Investigativas de los estudiantes.

\section{MÉTODOS Y MATERIALES}

Para alcanzar los objetivos propuestos, se direccionó a una investigación con enfoque cuantitativo de nivel explicativo con el objetivo de conocer la relación de las variables Aprendizaje Colaborativo y Competencias Investigativas, para la cual se aplicó un cuestionario para cada uno.

\section{RESULTADOS}

La investigación se apoyó del tratamiento estadístico, para ello se consideró el programa SPSS, con el cual se estableció la contratación de las hipótesis, los cuales se muestran a continuación:

\section{Prueba de hipótesis general}

HGA. El Aprendizaje Colaborativo se relaciona significativamente con las Competencias Investigativas de los estudiantes.

HGO. El Aprendizaje Colaborativo no se relaciona significativamente con las Competencias Investigativas de los estudiantes.

Tabla 1

Correlación de la hipótesis general

\begin{tabular}{lllr}
\hline \multicolumn{3}{c}{ Correlaciones } \\
\hline & & \multicolumn{1}{c}{$\begin{array}{l}\text { Competencias } \\
\text { Investigativas }\end{array}$} \\
\hline \multirow{3}{*}{$\begin{array}{l}\text { Rho de } \\
\text { Spearman }\end{array}$} & $\begin{array}{l}\text { Aprendizaje } \\
\text { Colaborativo }\end{array}$ & $\begin{array}{l}\text { Coeficiente de } \\
\text { correlación }\end{array}$ & 0,804 \\
\cline { 3 - 4 } & & Sig. (bilateral) & 0,000 \\
\cline { 3 - 4 } & & $\mathrm{N}$ & 128 \\
\hline
\end{tabular}

Con la fórmula de Rho de Spearman, margen de error al $5 \%$, se estableció un coeficiente de correlación muy buena de Rho = 0,804 y el $p=0,000$. Por lo cual se rechaza la hipótesis nula y se acepta la hipótesis alterna, que en base a lo manifestado por la muestra se entiende que el aprendizaje colaborativo es indiferente para incidir idóneamente en las competencias investigativas.

\section{Contrastación de la primera hipótesis específica}

H1. La Interdependencia Positiva se relaciona significativamente con las Competencias Investigativas de los estudiantes

Ho. La Interdependencia Positiva no se relaciona significativamente con las Competencias Investigativas de los estudiantes 
Tabla 2

Correlación de la primera hipótesis especifica

Correlaciones

\begin{tabular}{llrr}
\hline & & $\begin{array}{r}\text { Competencias } \\
\text { Investigativas }\end{array}$ \\
\hline & & $\begin{array}{l}\text { Coeficiente de } \\
\text { correlación }\end{array}$ & 0,401 \\
\cline { 3 - 3 } $\begin{array}{l}\text { Rho de } \\
\text { Spearman }\end{array}$ & Interdependencia & & \\
\cline { 3 - 4 } & Sig. (bilateral) & 0,000 \\
\cline { 2 - 3 } & $\mathrm{N}$ & 128 \\
\hline
\end{tabular}

Con el Rho de Spearman y un margen de error al 5\%, se halló un coeficiente de correlación moderada de Rho $=0.401$ y el $p=$ 0,000 . Por ello, se rechaza la hipótesis nula y se acepta la hipótesis alterna, que en referencia a lo señalado por la muestra se entiende que la interdependencia positiva es poco adecuada para incidir idóneamente en las competencias investigativas

\section{Contrastación de la segunda hipótesis específica}

H2. La Responsabilidad Individual y de Equipo se relaciona significativamente con las Competencias Investigativas de los estudiantes

Ho. La Responsabilidad Individual y de Equipo no se relaciona significativamente con las Competencias Investigativas de los estudiantes

Tabla 3

Correlación de la segunda hipótesis especifica

\begin{tabular}{lllr}
\hline \multicolumn{3}{c}{ Correlaciones } & \\
\hline & & $\begin{array}{l}\text { Competencias } \\
\text { Investigativas }\end{array}$ \\
\hline Rho de & $\begin{array}{l}\text { Responsabilidad } \\
\text { individual y de } \\
\text { equipo }\end{array}$ & $\begin{array}{l}\text { Coeficiente de } \\
\text { correlación }\end{array}$ & 0,613 \\
\cline { 3 - 4 } & Sig. (bilateral) & 0,000 \\
\cline { 3 - 4 } & $\mathrm{N}$ & 128
\end{tabular}

Se estableció una correlación buena de Rho $=0,613$ y el $p=$ 0,000 . Se acepta la hipótesis alterna, que en referencia a lo señalado por la muestra se señala que la responsabilidad individual y de equipo es indiferente para incidir idóneamente en las competencias investigativas.

\section{Contrastación de la tercera hipótesis específica}

H3. La Interacción Estimuladora se relaciona significativamente con las Competencias Investigativas de los estudiantes

Ho. La Interacción Estimuladora no se relaciona significativamente con las Competencias Investigativas de los estudiantes

\section{Tabla 4}

Correlación de la tercera hipótesis específica

\begin{tabular}{|c|c|c|c|}
\hline \multicolumn{4}{|c|}{ Correlaciones } \\
\hline & & & $\begin{array}{l}\text { Competencias } \\
\text { Investigativas }\end{array}$ \\
\hline \multirow{3}{*}{$\begin{array}{l}\text { Rho de } \\
\text { Spearman }\end{array}$} & \multirow{3}{*}{$\begin{array}{l}\text { Interacción } \\
\text { estimuladora }\end{array}$} & $\begin{array}{l}\text { Coeficiente de } \\
\text { correlación }\end{array}$ & 0,559 \\
\hline & & Sig. (bilateral) & 0,000 \\
\hline & & $\mathrm{N}$ & 128 \\
\hline
\end{tabular}

Se determinó una correlación moderada de Rho $=0.559$. En otras palabras se acepta la hipótesis alterna, que en referencia a lo manifestado por la muestra se entiende que la interacción estimuladora es indiferente para incidir idóneamente en las competencias investigatívas.

\section{DISCUSIÓN}

Este estudio científico permitió responder al objetivo general propuesto: Analizar la relación entre el Aprendizaje Colaboratívo con las Competencias Investigatívas de los estudiantes.

En la hipótesis general, se estableció una correlación muy buena de Rho $=0.804$, entre las variables Aprendizaje Colaboratívo y Competencias Investigatívas, cuyos resultados se deben a que en la primera variable el $58.6 \%$ los estudiantes señalan estar indiferentes, seguido del $38.3 \%$ de poco adecuado, mientras en la segunda variable el $58.1 \%$ de ellos alcanzan un nivel regular, seguidos del $41.4 \%$ en el nivel malo. Por ello, evidenciamos que la ausencia de mejoras en la forma en la que se aplica el aprendizaje colaborativo no fomenta el desarrollo de las competencias investigativas.

La primera hipótesis específica identificó una correlación moderada de Rho $=.401$, con respecto a la dimensión interdependencia positiva y la variable competencias investigativas, cuyas opiniones resaltan que la primera dimensión es poco adecuada para un $56.3 \%$ de estudiantes, seguido de un $31.3 \%$ que se muestran indiferentes; en tanto que las competencias investigativas les permitieron que el $58.1 \%$ alcancen el nivel regular, seguidos del $41.4 \%$ en el nivel malo. Con ello advertimos que los estudiantes aún no son capaces de poder trabajar en equipo de forma positiva, así como poder argumentar en los temas desarrollados en clase incidiendo así en las calificaciones poco destacadas de las competencias investigativas.

En cuanto al análisis de la segunda hipótesis específica se determinó una correlación buena de $\mathrm{Rho}=0,613$, que corresponde a la dimensión responsabilidad individual y de equipo y la variable competencias investigativas, cuyas opiniones mayoritarias destacan que en la mencionada dimensión el $60,9 \%$ de los estudiantes son indiferentes, lejos del $26,6 \%$ como poco adecuado; mientras que, en la variable en análisis, el $58,1 \%$ alcanzó el nivel regular, seguido del $41,4 \%$ en el nivel malo. Estableciendo que los estudiantes aún no cuentan con argumentos sólidos en el debate en clase, incluso no tienen presente a los autores que refuercen sus aprendizajes lo cual disminuye las posibilidades de mejorar las competencias investigativas.

En la tercera hipótesis específica se estableció una correlación moderada de Rho $=0,559$ que hace referencia a la dimensión interacción estimuladora y la variable competencias investigativas, cuyas opiniones resaltan que esta dimensión es indiferente para un $57,0 \%$ de estudiantes, seguido de un $39,8 \%$ que lo refieren de poco adecuado; mientras que las competencias investigativas les permitieron que el $58,1 \%$ alcancen el nivel regular, seguidos del $41,4 \%$ en el nivel malo. Con lo cual confirmamos que la ausencia de alternativas que incentiven el aprendizaje entre compañeros, dentro del espacio donde se desarrolla las clases impide que los conocimientos con respecto a las competencias investigativas sean evidentes.

Analizando estos resultados con los de Porcel (2016) quien detalla que el aprendizaje colaborativo en estudiantes universitarios suele alcanzar el nivel medio lo cual incide en el rendimiento académico con calificaciones medias, por ello indica que en los estudiantes se necesita promover, motivar, para que tomen conciencia de su propio aprender, los cuales guardan relación a lo encontrado a esta investigación en el cual notamos que la forma en la cual los grupos trabajan en las actividades académicas están relacionadas a las 
competencias investigativas. En otras palabras, el aporte de los integrantes en grupo es muy importante para reforzar los conocimientos de nivel superior demostrado en las calificaciones manifestados en la muestra de cada investigación.

En cuanto a las competencias investigativas, los resultados de Suárez (2013) dan cuenta que los aprendizajes deben ser visualizados desde una perspectiva multidimensional que incluyen: contenidos, procesos y condiciones, el cual debe ser visto como el proceso cooperativo, si bien el desarrollo de las competencias es progresivo, es necesario identificar metodologías que ayuden a que los aprendizajes sean más eficientes.

En base a todo lo analizado anteriormente podemos concluir que en la hipótesis general se determinó una muy buena correlación muy buena, que hace referencia a las repercusiones del aprendizaje colaborativo señalado mayoritariamente como indiferente, sobre las competencias investigativas cuyas calificaciones destacan por su nivel regular. Concluyendo que las actividades académicas en grupo no son aplicadas en base a las necesidades investigativas durante la formación de los estudiantes.

La primera hipótesis específica halló una correlación moderada, que indica el grado de incidencia presente en la interdependencia positiva como poco adecuado, con respecto a las competencias investigativas cuyas calificaciones son regulares, reflejados específicamente en el reconocimiento e identificación de los argumentos de investigación y el conocimiento de la ciencia. Resultando que el poco de optimismo en la argumentación científica en los grupos de trabajo disminuye la posibilidad de mejorar las calificaciones en referencia a las competencias investigativas.

La segunda hipótesis específica comprobó la existencia de una correlación buena, ya que la Responsabilidad individual y de equipo es considerado indiferente por la mayoría de estudiantes, lo cual poco eficiente en la relación con las competencias investigativas, particularmente en la interpretación de fenómenos científicos donde mayormente se manifestaron puntajes malos. Concluyendo que los estudiantes no trabajan idóneamente de forma grupal, puesto que no manejan una amplia bibliografía para poder argumentar en los trabajos requeridos en clase, perjudicando así al fortalecimiento de los conocimientos sobre el proceso de investigación.

La tercera hipótesis específica refiere que existe una correlación moderada, puesto que la interacción estimuladora es identificada como indiferente por la mayoría de la muestra, incidiendo inadecuadamente en las competencias investigativas, como en la reflexión de las implicancias científicas y tecnológicas cuyas calificaciones de este ítem es de malo. Entendiendo que el apoyo entre compañeros en cuanto a materiales académicos y motivación resultan necesarios para el aula disminuir la problemática en cuanto a comprensión y análisis del fenómeno científico y su proceso de análisis.

\section{AGRADECIMIENTOS}

Alos estudiantes, docentes de UNFV, por su aporte

\section{REFERENCIAS BIBLIOGRÁFICAS}

Álvarez, V. (2015). Aprendizaje colaborativo mediado por TIC en la enseñanza universitaria: un acercamiento a las percepciones y experiencias de profesores y alumnos de la Universidad Autónoma de Chihuahua (Tesis

Posgrado) Universidad de Salamanca, Salamanca, España. $R$ e c u p e $r$ a d o e $n$ : https://gredos.usal.es/jspui/bitstream/10366/127968/1/D DOMI_AlvarezOlivasV_MetodosAudiovisualesPedagogi a.pdf

Agencia Andina (2018). Falta de profesionales técnicos obligará a empresarios a importarlos, afirman. Recuperado en: https://andina.pe/agencia/noticia-faltaprofesionales-tecnicos-obligara-a-empresarios-aimportarlos-afirman-712139.aspx

INEI (2011). Informe Técnico: Evolución de la Pobreza 2007$2011 . R$ e c u p e r a d o e n : https://www.inei.gob.pe/media/cifras de pobreza/pobrez a informetecnico.pdf

Ley N³0512.(2016) Diario Oficial El Peruano, Lima, Perú, 2 de noviembre de 2016

Páez, J. (2016). Desarrollo de competencias investigativas basadas en la concepción sistémica de ambiente, en estudiantes de la escuela Normal Superior de Montería (Tesis de Posgrado) Universidad Nacional de Colombia. Medellín, Colombia. Recuperado en: http://bdigital.unal.edu.co/55895/1/desarrollocompetenci asinvestigativas.pdf

Porcel, T. (2016). Aprendizaje colaborativo, procesamiento estratégico de la información y rendimiento académico en estudiantes de la Facultad de Educación de la Universidad Nacional Amazónica de Madre de Dios, 2015 (Tesis Posgrado) UNMSM, Lima, Perú.

Diario Correo (2019). La falta de profesionales técnicos se mantiene en 200 mil. Recuperado en: https://diariocorreo.pe/economia/senati-faltaprofesionales-tecnicos-mantiene-200-mil-865701/

SINEACE (2015). Demanda laboral de técnicos en el Perú y expectativas sobre la certificación de competencias. Lima: SINEACE

Suárez, A. (2013). Estrategia pedagógica para el desarrollo de competencias investigativas en losdocentes de los Liceos del Ejército (Tesis Posgrado) Universidad Libre, $\mathrm{C}$ o I o m b i a. R e c u p e r a d o e $\mathrm{n}$ : https://repository.unilibre.edu.co/bitstream/handle/10901/ 8497/trabajo\%20sustentacioncd.pdf?sequence=1\&isAllo wed $=y$ 